\title{
A Systematic Survey, Taxonomic Study and Conservation of Genus Salacia (Celastraceae) in North Central Province of Sri Lanka
}

\author{
Senevirathne W.I.N.S.* and Hettiarachchi P.L. \\ Department of Biological Sciences, Rajarata University of Sri Lanka, Mihintale, Sri Lanka \\ *nirodha.sewwandi@gmail.com
}

\begin{abstract}
Genus Salacia is a climbing shrub comprised of nearly 200 species out of which, five have been reported in Sri Lanka. Salacia species are important components in many Ayurveda preparations and traditional medicine. Since $S$. reticultata and $S$. oblonga possess scientifically proven therapeutic benefits for an array of burning health issues, people tend to over exploit them from the wild to fulfill the existing demand. As a result, these two have become endangered in Sri Lanka. Therefore, recording existing populations in different agro-ecological regions with their abundance has become one of the key issues in conservation and sustainable use of these plants. Moreover, these species exhibit morphological plasticity and documented vegetative characters to differentiate one from the other are scares. The present study was launched to carry out a systematic survey and taxonomic study of different populations of Salacia species in the North Central province with the view of confirming taxonomic position of existing populations, documenting vegetative characters as there are overlapping morphological characters and flowering is seasonal and identifying unique populations for conservation. In the Systematic survey 28 localities out of which 15 are new records in the North Central Province where Salacia species were growing naturally were found. Representative samples were collected from twenty locations and identified, subsequently authenticated and used for the morphological study. Ninety morphological and anatomical characters of each population were scored by using standard methods. Methods for leaf clearing and wood maceration suitable for Genus Salacia were developed. A preliminary screening of phytochemicals was done using TLC. Leaf and stem extracts were obtained by cold extraction and Soxhlet method. TLC profiles were recorded under UV, using Anisaldehyde-sulphuric acid and Iodine vapour. Taxonomic relationships among collected samples were analyzed. Out of the ninety characters studied, fifty four could be identified as clear characters for species demarcation. Based on the study, eight samples could be clearly identified as $S$. reticulata, six as $S$. oblonga but the remaining six displayed a mixture of morphological characters of $S$. chinensis, S. reticulata and S. oblonga. The population collected from Ritigala (a new locality) showed deviation in characters from all others and hence need to draw the attention of conservationists.
\end{abstract}

Keywords: S. reticulata, S. oblonga, Taxonomic relationship

Proceedings of the International Forestry and Environment Symposium 2016, Department of Forestry and Environmental Science, University of Sri Jayewardenepura, Sri Lanka. 